\title{
Management of Isolated Lateral Malleolar Fracture
}

\author{
Ismail A. Yassin, Mohammed A. Hassan, Mohammed A. Sultan* \\ Department of Orthopedic Surgery,Faculty of Medicine, Al-Azhar University \\ *Corresponding author: Mohammed A. Sultan; Mobile: 01000799386; Email: sultan_28@yahoo.com
}

\begin{abstract}
Background: fractures of the lateral malleolus are the most common type of fracture. Various methods of lateral malleolar fixation have been utilized, all with acceptable results. Aim of the Work: to focus light on important modalities in management of isolated lateral malleolar fracture considering the advantages, the disadvantages and determine different protocol of management regarding the most recent guidelines for this group of patients. Patients and Methods: this study was conducted on twenty patients with fresh isolated lateral malleolar fracture. No or only minimal pain on the medial side was also a prerequisite, safeguarding against a ligamentous SE IV injury of the deltoid ligament. The patients were 4 men and 16 women with a mean age of 36 (22-50) years old. Results: all fractures united within an average time to union of 8.2 weeks. in all patients the average time to full weight bearing was 7.2 weeks. Most of complications were minor and resolved within 21 days. Conclusion: good anatomical reduction is essential for good clinical outcome irrespective of the type of fracture. Nevertheless, if an acceptable reduction cannot be achieved using closed techniques, one should proceed with open reduction and internal fixation without hesitation.
\end{abstract}

Keywords: isolated lateral malleolar fracture, management modalities

\section{Introduction}

There are many types of ankle fractures, and treatments vary significantly depending on the location and severity of the injury. Understanding what to do about a broken ankle requires some information about how these injuries occur. The ankle is a complex joint that forms where three bones come together. The bones of the lower leg, the tibia, and the fibula are above the joint, and the talus is below the joint ${ }^{(\mathbf{1})}$.

The tibia is the larger, weight-bearing bone of the lower leg. Of the weight transferred through the leg, about $90 \%$ is carried by the tibia. The fibula is the smaller bone on the outside of the leg. It only carries about $10 \%$ of your body weight. Both the tibia and the fibula wrap around the talus to form the ankle joint. The bony prominences at the ankle are called the medial malleolus (the end of the tibia) and the lateral malleolus (the end of the fibula) ${ }^{(1)}$.

The ends of these bones form a cup-shape that the talus bone sits within. The most important aspect of ankle fracture treatment is understanding how the talus moves in relation to the ends of the tibia and fibula. Fractures of the ankle are either stable (the movement of the talus is unchanged) or unstable (the talus does not move in a normal manner). This means that the joint is not held in a symmetric position--when the ankle fracture is unstable, more invasive treatment is needed (1).

When a broken ankle occurs, the injury may be to the end of the tibia (the medial malleolus) or to the fibula (the lateral malleolus), or both ${ }^{(\mathbf{1})}$.
Determining how to proceed with treatment depends on where the injury has occurred. There are many types of ankle fractures. Lateral malleolus fractures (fibula only) are the most common type of ankle fracture. Medial malleolus fractures (tibia only) are relatively uncommon. In general, a displaced (out of position) medial malleolus fracture is treated with surgery. Bimalleolar ankle fractures (both tibia and fibula) occur when there is an injury to both the inner and the outer side of the ankle. These injuries always result in an unstable ankle joint, and in most active patients, surgery will be recommended. Fractures of the posterior malleolus are generally found in association with bimalleolar ankle fractures in which case the injury is called a trimalleolar ankle fracture ${ }^{(\mathbf{1})}$.

In this study we focused on isolated fracture of the lateral malleolus. The lateral malleolus is the name given to the bone on the outside of the ankle joint. Fractures of the lateral malleolus are the most common type of fracture. These injuries typically occur when the ankle is either twisted or rolled, often with an awkward or uneven step. The good news is that most lateral malleolus fractures are considered stable ankle fractures and can be treated without surgery ${ }^{(2)}$.

Lateral malleolus fractures cause pain, swelling, and bruising around the ankle. Pain in other areas of the foot and ankle should be reason to suspect a more serious ankle injury than an isolated lateral malleolus fracture. Pain and swelling on the inner side of the ankle (along with a lateral malleolus fracture) may indicate the possibility of an injury 
called bimalleolar equivalent fracture--a specific type of unstable ankle fracture that may require surgery. Anyone with a lateral malleolus fracture should be examined for signs of a more serious, unstable ankle fracture ${ }^{(4)}$.

Unstable ankle fracture is likely to develop early ankle arthritis. The good news is that isolated lateral malleolus fractures are almost always stable ankle fractures, and therefore do not have this problem $^{(2)}$.

Treatment of Lateral Malleolus Fractures ice application: to reduce pain and minimize swelling. Elevationis important to keep swelling limited. Be sure your ankle is above your heart (in order to do this, you have to lie down, you can't do it sitting!). Nonsteroidal anti-inflammatory medications are helpful at controlling both swelling and pain. Rest/Immobilization: limiting weight-bearing help to control pain and swelling. Usually a week or two with crutches will control these symptoms ${ }^{(2)}$.

Surgery may be indicated if there is talar shift or $>3 \mathrm{~mm}$ displacement or with ipsilateral syndesmotic injury ${ }^{(3)}$.

\section{Aim of the Work}

This study is aiming to focus light on important modalities in management of isolated lateral malleolar fracture considering the advantages, the disadvantages and determine different protocol of management regarding the most recent guidelines for this group of patients.

\section{Patients and Methods}

Between November 2016 and October 2017, twenty patients with fresh isolated lateral malleolar fracture were included in a prospective manner. The study was started after ethical committee approval. An informed consent was signed after full explanation of the procedure for each parentis.

No or only minimal pain on the medial side was also a prerequisite, safeguarding against a ligamentous SE IV injury of the deltoid ligament. 20 patients (4 men and 16 women) with a mean age of 36 (22-50) years old. we managed 4 patients with below knee cast, 9 patients with butters plate 3 of them were introduce by the MIPO technique, 2 patients with tension band, 3 patients with intramedullary screw and 2 patients by lag screws only technique. The patients who had surgery were operated on in a supine position and under tourniquet control. Prophylactic antibiotic treatment was started prior to tourniquet application and continued for 48 hours postoperatively.

For the patients who were managed conservatively if severe swelling was present, the ankle was immobilized temporarily with a plaster of Paris (POP) back-slab, and elevated on several pillows. Then after about one week when edema subside we did blew knee cast. Materials (prepared before the procedure) are 2 rolls $150 \mathrm{~mm}$ padding, 5 plaster of Paris rolls, $150 \mathrm{~mm}$ wide, water, aprons to protect team members and patient. The plaster cast was applied on the hanging leg (knee flexed to 90 degrees and ankle held at 90 degrees). Padding was made thicker over the malleoli and around the fibular head. The cast reaches from the MTP joints of the toes to just below the knee joint, avoiding the top of the plaster being at the level of the neck of the fibula, where the common peroneal nerve crosses.

For the application of the lateral plate, a longitudinal incision was made on the fibula. The plate was placed with attention paid to the neurovascular structures, and generally fixed with 3.5 sized cortical screws; $3-4$ screws to the proximal of the fracture and 2-3 to the distal.

When using (MIPO) technique, a small incision was planned over the subcutaneous surface of the proximal fibula or directly on the fracture. Through this window a $10 \mathrm{~mm}$ curved osteotome was passed subperiosteally to make a tunnel. Through this tunnel plate was inserted under c-arm guidance.

For the tension band technique, a short longitudinal incision was made over the fibula, extending from the fracture line to the tip of the fibula. Being careful of the neurovascular structures, the fracture was reduced and temporarily fixed with a single clamp. In all patients, $2 \mathrm{~K}$-wires and cerclage wire were used as fixation materials. The 2 parallel $\mathrm{K}$-wires were driven intramedullary from the fibular tip, and from the level of the fracture, the wires were advanced at least double the length of the fracture line from the fibular tip. Generally, K-wires of 1.8 $\mathrm{mm}$ or $2 \mathrm{~mm}$ thickness were used to approximately fill the proximal intramedullary canal. Immediately proximal to the fracture, a hole was opened in the fibula with a 3.2 sized drill from anterior to posterior, protecting the posterior anatomic structures. From this hole, the cerclage wire was passed medially to the K-wires in patients with poor bone quality, and in patients with good bone quality, it was passed lateral to the K-wires. In the distal, it was passed medial to the K-wires, the reduction clamp was removed and the cerclage wire was tied in a figureof-eight. Then, by bending the ends of both K-wires, the ends were laid over the fibular tip, turned towards the talofibular joint, so as not to create any irritation.

For lag screw only fixation, the fibular fracture morphology needs to be of a simple oblique or spiral pattern with no bony comminution at the fracture site. In addition, the fracture itself must to be of sufficient length to accept at least two screws placed $1 \mathrm{~cm}$ apart and perpendicular to the fracture line. The surgical technique adopted by the unit for lag screw fixation was that recommended by the 
AO/ASIF group: lateral incisions were placed over the anterior edge of the fibula at the fracture site, and soft tissue dissection is kept to a minimum. The fracture was reduced anatomically, and two or three stainless steel lag screws, typically either partially threaded cancellous screws or $3.5-\mathrm{mm}$ cortical screws in lag mode, were inserted in the anteriorposterior direction. Osteoporosis is a relative contraindication to this method of fracture treatment, so we have not treated any patient over 55 years of age with lag screw only fixation.

For intramedullary screw fixation, a lateral malleolus fracture must be simple or minimally comminuted because intramedullary fixation will not reliably maintain length in comminuted fractures. Ideally, the lateral malleolus fracture should be of a transverse or short oblique configuration. The technique involves using image intensification to aid anatomic reduction by inverting the foot and then achieving and maintaining fracture reduction with a towel clipper cutaneously (This technique may be more difficult in a markedly obese or swollen ankle). While the fracture is stabilized, a stab incision was made just distal to the tip of the lateral malleolus, and a hole was made in the tip of the lateral malleolus with a $2.5-\mathrm{mm}$ drill bit trying to be somewhat posterior on the lateral malleolus tip (avoid anterior insertion). A $3.5-\mathrm{mm}$, fully threaded, self-tapping bone screw is then passed across the fracture site and into the proximal fibular fragment. The screw is tightened until its head reaches the bone. It is not possible to make more compression at the fracture site by further tightening of the screw because it is a fully threaded screw and serrations were on both sides of fracture site. Electric power drill can be used to insert the screw to give the screw the enough power to be bent with the long axis of distal fibula. The length of the screw varies between $100 \mathrm{~mm}$ and $120 \mathrm{~mm}$, depending on the fracture location and pattern.

The patients were operated on either by orthopedic consultants or junior doctors under the direct supervision of those consultants. All patients were followed after 1 and 6 weeks and after 3 months; $\mathrm{x}$-rays of the ankle were obtained on the $1^{\text {st }}$ week and after 6-12 weeks. At each examination the ankle circumference was measured. Range of motion was recorded in the weight-bearing position using a goniometer and was compared to the normal ankle. SPSS program was used and t-test was done.

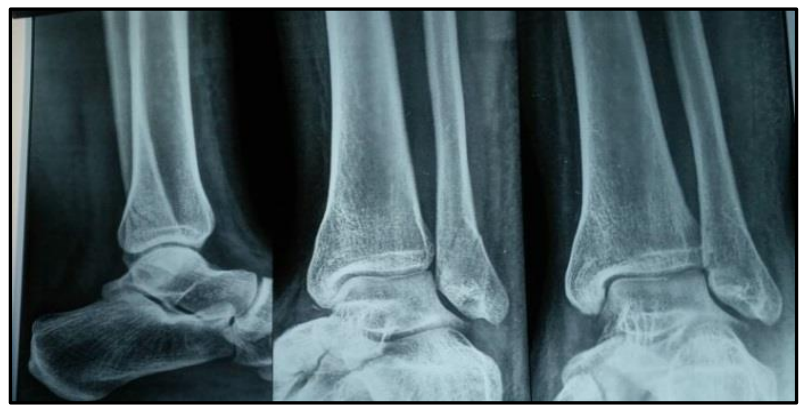

Figure (1): $1^{\text {st }}$ day after the injury X-ray.

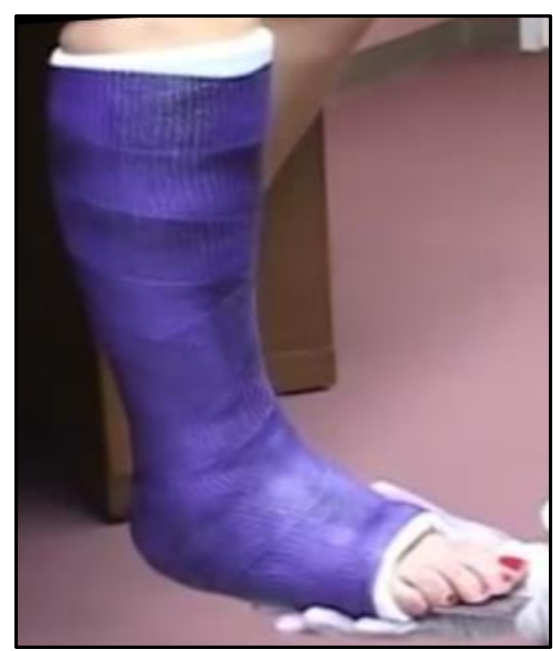

Figure (2): A blew knee cast was done on the 1st day after the injury.

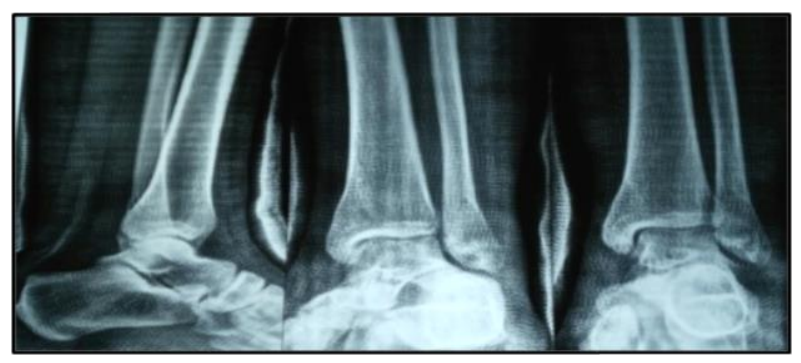

Figure (3): X-rays after 4 weeks (AP- Mortis Lateral views).

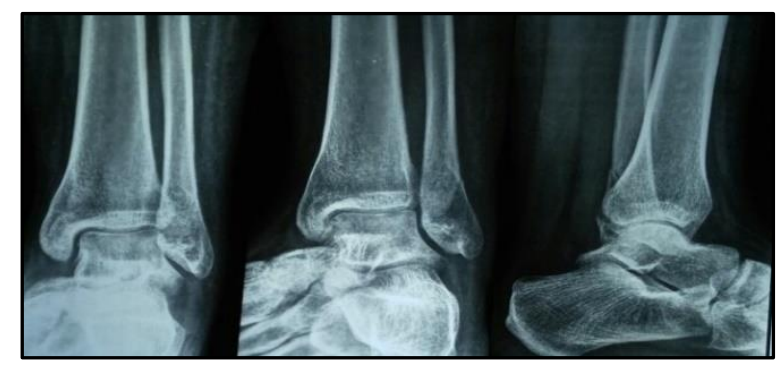

Figure (4): X-rays after 6 weeks (AP- Mortis Lateral views). 


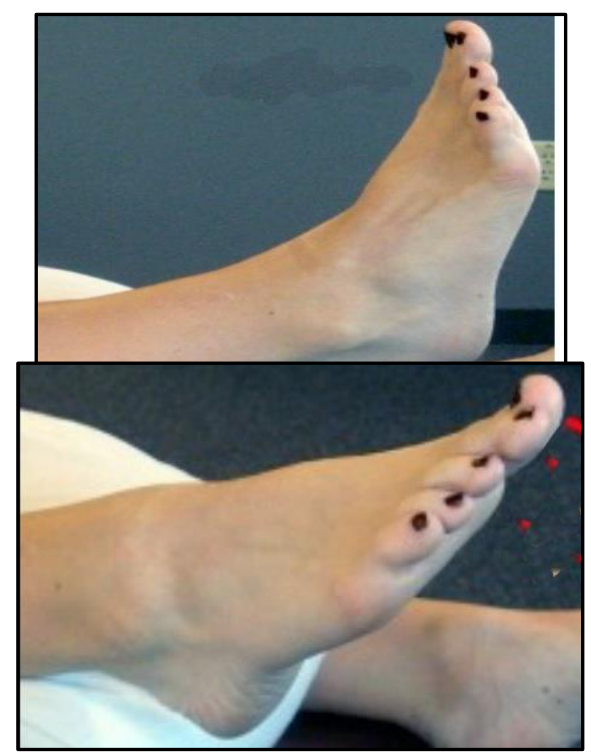

Figure (5): Painless full range of motion after 9 weeks.

\section{Results}

The mechanism of injury (Table 1) in the 20 patients included in this study were falls (40\%), motor vehicle accidents (35\%), motorcycle crashes (15\%), and direct blows (10\%). Evaluation of immediate postoperative $\mathrm{x}$-ray images for adequacy of reduction (Table 2) revealed a good reduction in 18 of $20(90 \%)$ patients, a fair reduction in 2 of 20 (10\%) patients, (Table 3). The average time to union was 8.2 weeks.

Duration of non-weight bearing mobilization varied from 6 to 10 weeks, with an average of 8.8 weeks. Time to full weight bearing ranged from 3 to 12 weeks, with an average time to full weight bearing of 7.2 weeks. There was a $100 \%$ rate of union for all lateral malleolus fractures in this group.

No deep wound infections occurred. No patient has complained of tenderness or prominence of the hardware and no patient has complained of impingement symptoms.

A functional rating scale (Table 4) was used to subjectively evaluate ankles in the patients who continued with clinic appointments or could be contacted by phone or internet. Of these patients (Table 5), 11 patients (55\%) had an excellent result and $8(40 \%)$ were considered to have a good result. One patient (5\%) who had a fair functional rating had a shortened lateral malleolus $(2 \mathrm{~mm})$.Patients were evaluated for anatomic healing and not for long-term results related to degenerative arthritis.

Table (1): Mechanism of injury

\begin{tabular}{||l|c|c||}
\hline \multicolumn{1}{|c|}{ Mechanisms } & Number of patients & Percentage \\
\hline \hline Fall & 8 & 40 \\
Motor vehicle & 7 & 35 \\
Motorcycle & 3 & 15 \\
Direct blow & 2 & 10 \\
\hline Total & 20 & \\
\hline \hline
\end{tabular}

Table (2): Radiographic criteria.

\begin{tabular}{||l|l||}
\hline Good & Fibula out to length \\
& $<2 \mathrm{~mm}$ of posterior displacement \\
& $<1 \mathrm{~mm}$ increase in medial clear space \\
\hline Fair & Fibula shortened $<2 \mathrm{~mm}$ \\
& $2-4 \mathrm{~mm}$ of posterior displacement \\
& $1-3 \mathrm{~mm}$ increase in medial clear space \\
\hline Poor & Fibula shortened $>2 \mathrm{~mm}$ \\
& $>4 \mathrm{~mm}$ of posterior displacement \\
& $>3 \mathrm{~mm}$ increase in medial clear space \\
\hline \hline
\end{tabular}

Table (3): Reduction on x-ray

\begin{tabular}{||l|c|c||}
\hline \multicolumn{1}{|c|}{ Rating } & Number of patients & Percentage \\
\hline \hline Good & 18 & 90 \\
\hline Fair & 2 & 10 \\
\hline Poor & 0 & 0 \\
\hline Total & 20 & \\
\hline
\end{tabular}

Table (4): Functional rating scale (compared with normal ankle).

\begin{tabular}{||l|l||}
\hline Excellent & Normal range of motion without pain or stiffnessand return to previous activity level \\
\hline Good & $\begin{array}{l}\text { Normal range of motion without stiffness, return to previous activity level with only occasional pain } \\
\text { following activity }\end{array}$ \\
\hline Fair & Decreased range of motion, frequent pain following activity \\
\hline Poor & Decreased range of motion, pain and stiffness at rest \\
\hline
\end{tabular}


Table (5): Functional results.

\begin{tabular}{|l|c|c|}
\hline \multicolumn{1}{|c|}{ Rating } & Number of Patients & Percentage \\
\hline \hline Excellent & 11 & 55 \\
\hline Good & 8 & 40 \\
\hline Fair & 1 & 5 \\
\hline Poor & 0 & 0 \\
\hline Total & 20 & \\
\hline
\end{tabular}

\section{Discussion}

Ankle fractures are common, with an incidence of approximately 130 fractures per 100,000 persons ${ }^{(4)}$. Conservative treatment (blew knee cast) of undisplaced or minimally displaced fractures has been shown to be successful. The importance of stable, anatomic fixation of the lateral malleolus fragment is highly appreciated to achieve satisfactory results in ankle fractures. Various methods of lateral malleolar fixation have been utilized, all with acceptable results (5).

The most common method of fixation of lateral malleolar fractures is the buttress plating. It provides stable fixation and maintains length of the lateral malleolus. It is preferred in cases where the malleolus is comminuted, as length could not be reliably maintained with an IM screw, in compound fractures and fractures with syndesmotic injury where syndesmotic screw needed (Weber type C). The complications of plate fixation of the lateral malleolus are wound healing, especially in swollen ankles and painful, prominent hardware often develop late ${ }^{(6)}$.

Minimum incision percutaneous plate osteosynthesis (MIPPO) is a simple procedure and no special instruments are required. The surgical exposure is much less and especially incision is avoided at distal fibula, where the plate exposure is common in open fixations. The procedure has advantage of early fracture healing and devoid of complications like devitalisation of skin and plate exposure and further the procedure is simple and does not need any special equipment ${ }^{(7)}$.

Tension band wiring with $2 \mathrm{~K}$-wires and cerclage wire requires a shorter incision and cause less soft tissue damage as in posterior plating, without irritating the peroneal tendons. This effective fixation method whichis mostly used for medial malleolar fractures may also be alternative for lateral malleolar fractures ${ }^{(\mathbf{8 , 9})}$.

During lateral malleolar fracture surgery, the peroneal nerve which penetrates the crural fascia at a mean of $5 \mathrm{~cm}$ proximal to the level of the ankle joint and its branch intermediate dorsal cutaneous nerve may be damaged ${ }^{(\mathbf{1 0}, \mathbf{1 1})}$.

Wide variations are seen at the emergence site of the lateral compartment of the superficial peroneal nerve from the crural fascia. In a study by Mirza $e t$ al. ${ }^{\left({ }^{(2)}\right.}$ this nerve was found to be mean $11.6 \mathrm{~cm}$ from the tip of the lateral malleolus. Thus, a long incision increases the possibility of nerve injury. As both treatment methods appear to be evenly effective, tension band technique which can be applied cheaply with universally available may have a significant advantage over plate and screw technique ${ }^{(\mathbf{1 2})}$.

Lag screw only fixation of fibular fractures is an attractive alternative to plate osteosynthesis. Performing an open reduction allows the surgeon to achieve the anatomical reduction shown to be integral to a good outcome ${ }^{(\mathbf{1 3})}$.

It also permits direct visualisation of the fracture personality, avoiding the inappropriate application of the technique of limited internal fixation and its complications ${ }^{(14)}$.

We feel this represents a direct advantage over other less invasive forms of fixation that have been described, such as percutaneous intramedullary fixation either using a screw or Knowles pin ${ }^{(\mathbf{1 5})}$.

While limited fixation is biomechanically weaker than plate osteosynthesis, the absence of any loss of fracture reduction in the present series suggests that while not absolutely rigid, fracture fixation is clearly stable. We have found no evidence to suggest that this less rigid fixation compromises fracture healing or clinical outcome. A potential disadvantage of less rigid fixation is the necessity for cast immobilization following surgery ${ }^{(16)}$.

Percutaneous screw fixation of lateral malleolus is also an acceptable technique for lateral malleolar fracture fixation. Covino et al. ${ }^{(17)}$ (in their retrospective clinical review and a biomechanical evaluation of lateral malleolus fixation) they noted no significant difference between interfragmentary versus plate fixation. They believed interfragmentary fixation avoided possible complications of lateral hardware and 
Ismail Yassin et al.

eliminated the possibility of screw penetration into the ankle joint.

Intramedullary fixation of lateral malleolus fractures provides stable fixation without prominent subcutaneous hardware. The Intramedullary position of the hardware reduces torque and bending moment on the device. Intramedullary fixation of weight bearing long bone fractures has largely replaced compression plating because of these biomechanical advantages. The advantages are also evident in the treatment of non-comminuted lateral malleolus fractures, and the technique should be utilized in indicated cases. Intramedullary fixation provides stable fixation, allowing early mobilization in this series. Bankston et al. (18) they found the intramedullary screw provided $66.5 \%$ of the strength of native bone compared to the lateral buttress plate provided $61.5 \%$. This was not statistically significant, but it did prove that an intramedullary screw provides stable fixation, thereby assuring that rehabilitation can be instituted early without the risk of loss of reduction. If reduction of the fracture can be achieved in a closed fashion (with the aid of image intensification), we believe that fixation of a lateral malleolus fracture may be performed with an axial Intramedullary screw percutaneously. Our closed technique utilizes minimal soft tissue dissection, thus decreasing the incidence of wound complications and painful hardware sites that are occasionally observed after open reduction and plating techniques. Other advantages include improved healing and shortened rehabilitation time because the fracture hematoma is not violated. We have initially selected only transverse or short oblique fractures as the primary candidates for this percutaneous technique. However, as our experience grows, perhaps other fracture configurations can also be treated by this method ${ }^{(\mathbf{1 8})}$.

Nevertheless, if an acceptable reduction cannot be achieved using this closed technique, one can and should proceed with open reduction and internal fixation without hesitation and without anything lost in the process.

Finally, use of more rigid subjective and objective criteria would allow more specific conclusions to be drawn about the patients' longterm functional results with different types of isolated malleolar fractures and its various techniques of fixation.

\section{Conclusion}

Good anatomical reduction is essential for good clinical outcome irrespective of the type of fracture. Nevertheless, if an acceptable reduction cannot be achieved using closed techniques, one should proceed with open reduction and internal fixation without hesitation.

\section{References}

1. Brett Crist MP and Stuart Fischer J (2013): Ortho Info. Retrieved from Ankle Fractures (Broken Ankle). American Academy of Orthopedic Surgeons., Available at: http://www.orthoinfo.aaos.org/topic.cfm?topic $=$ $\underline{\mathrm{A} 00391}$

2. Scott M Koehler, M (2016): Overview of ankle fractures in adults. Retrieved from up to date: http://www.uptodate.com/contents/overview-ofankle-fractures-inadults? source $=$ search_result\&search=Lateral+ Malleolus+Fracture \&selectedTitle $=1 \sim 150$.

3. Paul J (2017): Ankle Fracture Surgery. Retrieved from foot education: http://www.footeducation.com/page/anklefracture-surgery.

4. Bucholz RW, Heckman JD, Court-Brown C et al. (2006): Rockwood and Green's Fractures in Adults, 6th ed. Philadelphia: Lippincott Williams \& Wilkins, Pp. 212-233.

5. Bauer M (1985): Thiry years follow up of ankle fracture. Acta Orthop Scand., 56(2):103-6.

6. Schaffer JJ and Manoli A (1987): The Antiglide Plate for Distal Fibular Fixation. A Biomechanical Comparison with Fixation with a Lateral Plate. The Journal of Bone \& Joint Surgery, 69(4): 596-604.

7. Carlile GS and Giles NC (2011): Article name--. Foot and Ankle Surgery, 17(3): 119-123.

8. Ostrum RF andLitsky AS (1992): Tension band fixation of medial malleolus fractures. $\mathbf{J}$ Orthop Trauma, 6:464-8. 5.

9. Kanakis TE, Papadakis E, Orfanos A, Andreadakis A andXylouris E (1990): Figure eight tension band in the treatment of fractures and pseudarthroses of the medial malleolus. Injury, 21:393-7.

10. Kim HJ, Oh JK, Oh CW, Hwang JH and Biswal S (2010): Anterior transposition of the superficial peroneal nevre branch during the internal fixation of the lateral malleolus. J Orthop Trauma, 68:421-4. 
11. Olcay E, Ozturk A, Aksoy B, Ari Z, Bulbul M, Sahinoglu K (1998): Surgical anatomy and safe zones of the superficial peroneal branch of the peroneal nerve in the ankle. [Article in Turkish] Acta Orthop Traumatol Turc., 32:340-3.

12. Mirza A, Moriarty AM, Probe RA, Ellis TJ (2010): Percutaneous plating of the distal tibia and fibula: Risk of injury to the saphenous and superficial peroneal nerves. J Orthop Trauma, 24:495-8.

13. Thordarson DB, Motamed S, Hedman T, Ebramzadeh E and Bakshian S (1997): The effect of fibular malreduction on contact pressures in an ankle fracture malunion model. $\mathbf{J}$ Bone Joint Surg Am., 79:1809-1815.

14. Olerud C, Molander H, Olsson T, Hagstedt B (1986): Ankle fractures treated with nonrigid internal fixation. Injury, 17:23-27.
15. Lee YS, Huang CC, Chen CN, Lin CC (2005): Operative treatment of displaced lateral malleolar fractures: the Knowles pin technique. J Orthop Trauma, 19:192-197.

16. Weber $M$ and Krause $F$ (2005): Peroneal tendon lesions caused by antiglide plates used for fixation of lateral malleolar fractures: the effect of plate and screw position. Foot Ankle Int., 26:281-285.

17. Covino BM, Barsanti CM, Wolfe J and Wang GJ (1990): Internal Fixation of Lateral Malleolus Fractures: A Clinical and Biomechanical Comparison of Two Techni- ques. Orthopaedics Transaction Journal, 5: 90.

18. Bankston $A B$, Anderson $L D$ and Nimityongskul P (1994): Intramedullary Screw Fixation of Lateral Malleolus Frac- tures, Foot \& Ankle International, 15(11):. 599-607. 